symptoms of HIV positive and HIV negative MSM with LGV proctitis.

This is a cross-sectional study of MSM who tested positive for LGV attending our large STI centre in Brighton, UK between January 2013 and December 2019. We collected data on age, number of recent sexual partners, ano-rectal symptoms, HIV status, use of HIV Pre-exposure prophylaxis (PrEP) and recreational drug use.

49 MSM had a positive rectal LGV PCR between 20132019. The median age was 45 years ( $\mathrm{IQR}=39-53), 44(90 \%)$ of the MSM tested were symptomatic and the remaining five were asymptomatic. Eleven (22\%) reported recent chemsex, $35(71 \%)$ were HIV seropositive and six(43\%) of the 15 HIV negative MSM were using PrEP. The symptoms described by the symptomatic MSM were: rectal pain[27,(61\%)], rectal bleeding[21,(54\%)], rectal discharge[23,(52\%)], diarrhoea[8, $(18 \%)]$, tenesmus[6,(15\%)] and constipation[5,(13\%)]. There were no significant differences between the characteristics and presenting symptoms between HIV positive and negative MSM.

The symptoms of MSM diagnosed with LGV proctitis are varied and there were no differences between HIV positive and negative MSM. The heterogeneity of presenting symptoms of MSM with LGV proctitis leads to missed opportunities for diagnosis, increased morbidity due to delays in diagnosis and implications for onward transmission particularly where clinicians are not so familiar with LGV or do not have access to testing. Increasing awareness of the heterogeneity of the presentation of LGV in MSM may improve the diagnosis and the impact of public health campaigns to control LGV.

\section{P029 PHARYNGEAL CHLAMYDIA TRACHOMATIS IN HIV POSITIVE AND HIV NEGATIVE MEN WHO HAVE SEX WITH MEN (MSM)}

${ }^{1} \mathrm{~K}$ Samarasekara, ${ }^{1} \mathrm{C}$ Fitzpatrick, ${ }^{1} \mathrm{~F}$ Finnerty, ${ }^{1,2} \mathrm{D}$ Richardson*. 'Brighton and Sussex University Hospitals Nhs Trust, Brighton, UK; ${ }^{2}$ Brighton and Sussex Medical School, Brighton, UK

\subsection{6/sextrans-2021-sti.176}

Background Simultaneous testing for both Gonorrhoea and Chlamydia using dual nucleic acid amplification tests (NAAT) has increased pharyngeal Chlamydia testing in MSM. There has been increasing interest in pharyngeal Gonorrhoea due to emerging Gonorrhoea antimicrobial resistance but less is understood about the characteristics of pharyngeal Chlamydia in MSM.

Methods We aimed to explore the prevalence and associated features of pharyngeal Chlamydia amongst HIV positive and negative MSM in a large urban population. We collected data on HIV status, pharyngeal symptoms, number of recent sexual partners, use of HIV pre-exposure prophylaxis (PrEP), concomitant STIs, previous history of syphilis and current smoking status. Statistical analysis was performed using bivariate odds ratio and Mann Whitney $\mathrm{U}$ tests and 95\% confidence intervals.

Results In 2019, 6613 MSM attended for pharyngeal STI testing. $75 / 6613(1.13 \%, 95 \% \mathrm{CI}=0.9-1.14)$ tested positive for pharyngeal Chlamydia. The median age was 35 years $(\mathrm{IQR}=28-43)$, the median number of sexual partners in the preceding 3 months was three, four $(5 \%)$ reported throat symptoms, 22(29\%) were current smokers, 20(26\%) were HIV positive, and 24(44\%) of the HIV negative MSM were using PrEP. MSM with pharyngeal Chlamydia often had multi-site infection; rectal chlamydia[39(52\%)], urethral chlamydia[12 (16\%), and concomitant infection; early syphilis[2(3\%)] and gonorrhoea at any site[14(19\%). Twenty two(29\%) had previously treated syphilis. HIV positive MSM with pharyngeal Chlamydia were significantly more likely to have had previous syphilis $(\mathrm{OR}=4.9,95 \% \mathrm{CI} 1.6-14.7, \mathrm{p}=0.005)$ and were significantly older $(\mathrm{p}=0.02)$ than HIV negative MSM.

Conclusion We have shown that the prevalence of pharyngeal Chlamydia is $1.13 \%$ in MSM and only 5\% reported pharyngeal symptoms, similar to other studies. HIV positive MSM with pharyngeal Chlamydia were older and more likely to have had previous syphilis. Further research is needed to explore the characteristics of pharyngeal Chlamydia and the benefits of increased screening for asymptomatic pharyngeal Chlamydia in MSM.

\section{P030 HIV POST-EXPOSURE PROPHYLAXIS TO PRE-EXPOSURE PROPHYLAXIS TRANSITIONING IN MEN WHO HAVE SEX WITH MEN}

${ }^{1,2} \mathrm{D}$ Richardson*, ${ }^{1} \mathrm{~K}$ Nichols, ${ }^{1} \mathrm{~A}$ Pickering, ${ }^{1} \mathrm{~J}$ Devlin, ${ }^{1} \mathrm{Z}$ Buss, ${ }^{1} \mathrm{C}$ Fitzpatrick, ${ }^{1,3} \mathrm{~F}$ Cresswell. ${ }^{1}$ Brighton and Sussex University Hospitals Nhs Trust, Brighton, UK; ${ }^{2}$ Brighton and Sussex Medical School, Brighton, UK; ${ }^{3}$ London School of Hygiene and Tropical Medicine, London, UK

\subsection{6/sextrans-2021-sti.177}

Background HIV post-exposure prophylaxis following sexual exposure (PEPSE) and pre-exposure prophylaxis (PrEP) are used by people at sexual risk of HIV acquisition including men who have sex with men (MSM). Owing to the high risk of HIV seroconversion due to on-going risk behaviours, it is becoming commonplace for HIV-negative MSM requiring PEPSE (e.g. HIV sexual-exposure within 72-hours) to transition immediately to PrEP following the 28-days of PEPSE. We aimed to review how frequently PrEP is discussed and used by MSM following PEPSE.

Method We reviewed the electronic notes of MSM who had accessed PEPSE between January 2018 - November 2020 and collected information on sexual assault, recreational drug use at the time of PEPSE initiation, whether direct transition to PrEP had been discussed, and if PrEP had been initiated after PEPSE.

Results During the study period, 277 MSM accessed PEPSE. The median age was 32 years (IQR 26-43), 17 (6\%) started PEPSE following a sexual assault, $36(13 \%)$ were using recreational drugs during sex and $30(11 \%)$ had used PrEP previously. Discussion about direct transition to PrEP after PEPSE was documented in 155 (56\%) MSM, including 128 (51\%) MSM who had never used PrEP before, and 67 (24\%) MSM actually transitioned directly from PEPSE to PrEP. Clinicians were more likely to discuss PEPSE to PrEP transition in MSM with prior PrEP use $(\mathrm{p}<0.0001)$, and MSM were more likely to transition to PrEP from PEPSE if they had used PrEP previously $(\mathrm{p}<0.00001)$.

Conclusion Only 56\% of MSM who used PEPSE had a documented discussion about transitioning to PrEP and only a 24\% actually transitioned from PEPSE to PrEP. Where indicated, MSM who access PEPSE should have a documented discussion and ideally transition immediately onto PrEP following completion of PEPSE. 